\title{
EUS-Guided Pancreatic Duct Drainage for Repeat Pancreatitis in a Patient with Pancreatic Cancer
}

\author{
Ken Kamata Mamoru Takenaka Kosuke Minaga Toshiharu Sakurai \\ Tomohiro Watanabe Naoshi Nishida Masatoshi Kudo \\ Department of Gastroenterology and Hepatology, Kindai University School of Medicine, Osaka-Sayama, Japan
}

\section{Keywords}

Pancreatic cancer · Endoscopic ultrasonography ·

Pancreatitis

\begin{abstract}
Endoscopic ultrasound-guided pancreatic drainage (EUSPD) was performed in a patient with unresectable pancreatic cancer who developed pancreatitis. In this case, EUS-PD was useful as salvage therapy for pancreatitis as the transpapillary approach was difficult.

๑) 2017 S. Karger AG, Basel
\end{abstract}

Endoscopic ultrasound-guided pancreatic drainage (EUS-PD) has been described as a new drainage technique after unsuccessful transpapillary approach for pancreatic duct strictures [1-3]. Our patient was a 74-year-old man with unresectable pancreatic cancer receiving chemotherapy. Due to repeated pancreatitis, however, he could not continue treatment and was forced to stop chemotherapy. The patient had no history of chronic pancreatitis but abdominal CT showed a pancreatic duct stone in the main pancreatic duct at the pancreatic body. The main pancreatic duct was slightly dilated

\section{KARGER}

(C) 2017 S. Karger AG, Basel

E-Mail karger@karger.com

www.karger.com/ocl from the tail side of the stone, with localized pancreatitis observed around the pancreatic tail (Fig. 1). The pancreatic cancer was located in the pancreatic head, whereas the pancreatic stone was located in the pancreatic body (Fig. 1). After pancreatitis was resolved, transpapillary pancreatic duct drainage was attempted, but the guide wire could not be deployed over the pancreatic duct stricture caused by the tumor. Then, EUS-PD was performed to prevent pancreatitis induced by a pancreatic duct stricture. The patient was placed under sedation with a bolus infusion of $0.05 \mathrm{ml} / \mathrm{kg}$ propofol. The dilated pancreatic duct from the tail side of the stone, as viewed echoendoscopically (GF-UCT260; Olympus) from the stomach position, was punctured with a 19-gauge needle (Sonotip; Medi-Globe), with correct puncturing confirmed with contrast medium (Fig. 2). A 0.025-inch guidewire (VisiGlide2; Olympus) was inserted toward the pancreatic tail. After dilating the fistulous tract using a diathermic dilator, a 7-Fr plastic stent (7 cm in length; Boston Scientific) was successfully deployed (Fig. 3). There were no complications during the procedure. Seven days later, the patient's serum amylase concentration had decreased to 88 $\mathrm{U} / \mathrm{L}$ and he started dietary intake. One month after the procedure, he resumed chemotherapy, with no recurrence of pancreatitis to date. Findings in this patient in-

Dr. Mamoru Takenaka

Department of Gastroenterology and Hepatology Kindai University Faculty of Medicine

377-2 Ohno-Higashi, Osaka-Sayama, Osaka 589-8511 (Japan)

E-Mail mamoxyo45@gmail.com 


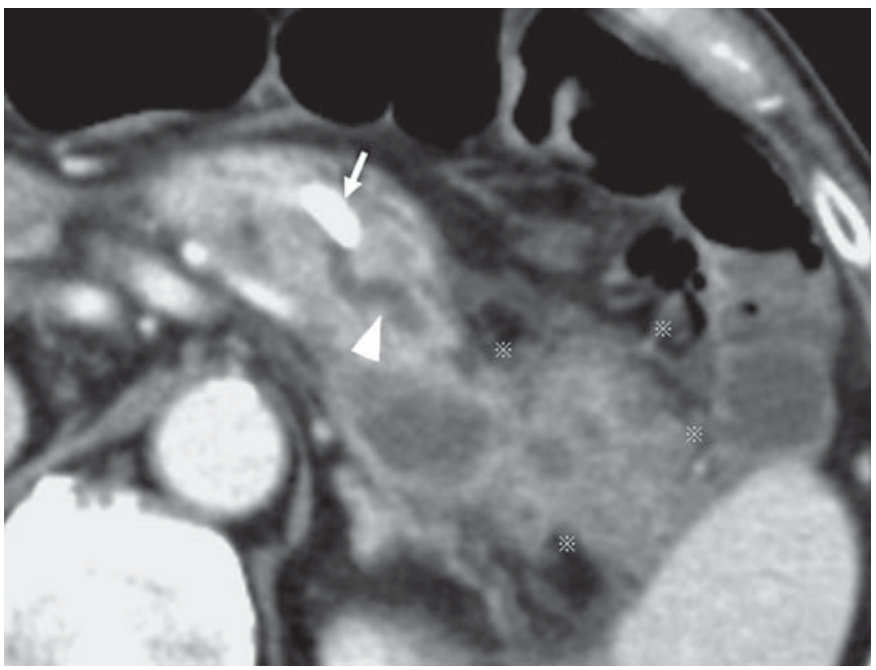

Fig. 1. Abdominal CT image of the patient, showing dilation of the main pancreatic duct (arrowhead) from the stone (arrow) to the tail side with localized pancreatitis $(※)$ observed around the pancreatic tail.

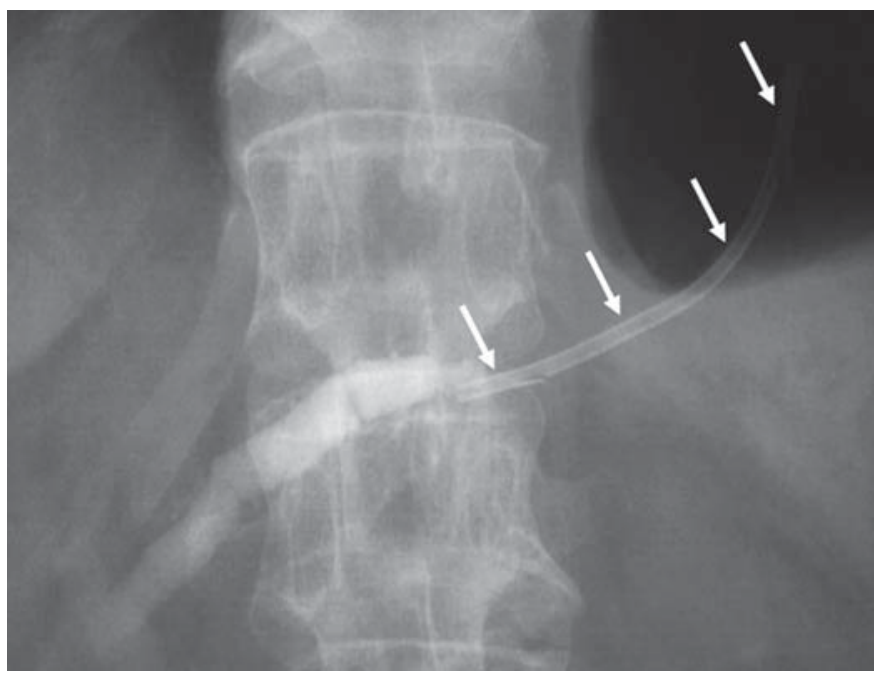

Fig. 3. Fluoroscopic image showing the deployment of a 7-Fr plastic stent (arrows) at the tail side from the stone between the pancreatic duct in the body of the pancreas and the gastric body.

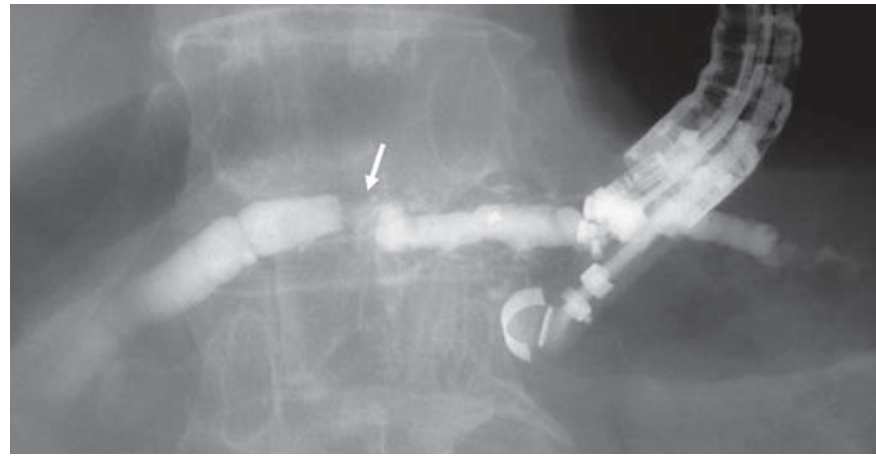

Fig. 2. Fluoroscopic image showing that the defect was a pancreatic stone (arrow) in the pancreatic body.

dicated that the pancreatic duct stone caused repeat pancreatitis, resulting in discontinuation of chemotherapy. Although transpapillary pancreatic duct drainage was considerable, pancreatic duct drainage over the tumor and the pancreatic duct stricture was limited. The presence of the tumor in the head of the pancreas may have made stone removal more difficult. EUS-PD may constitute salvage therapy when the transpapillary approach is difficult.

\section{Disclosure Statement}

All authors disclosed no financial relationships relevant to this publication.

\section{References}

1 Itoi T, Kasuya K, Sofuni A, et al: Endoscopic ultrasonography-guided pancreatic duct access: techniques and literature review of pancreatography, transmural drainage and rendezvous techniques. Dig Endosc 2013;25:241-252

2 Kurihara T, Itoi T, Sofuni A, et al: Endoscopic ultrasonography-guided pancreatic duct drainage after failed endoscopic retrograde cholangiopancreatography in patients with malignant and benign pancreatic duct obstructions. Dig Endosc 2013;25(suppl 2):109-116.

3 Tyberg A, Sharaiha RZ, Kedia P, et al: EUS-guided pancreatic drainage for pancreatic strictures after failed ERCP: a multicenter international collaborative study. Gastrointest Endosc 2017;85:164-169. 